\title{
Nouvelles synthèses de pyrimidines
}

par

\author{
E. Cherbuliez et K. N. Stavriteh.
}

18. II. 22.)

En soumettant la méthylène-asparagine (I) à la réaction de Hofmann (traitement à l'hypobromite) dans le but de la transformer en un dérivé de l'acide diamino-propionique, nous avons obtenu un acide halogéné $\mathrm{C}_{5} \mathrm{H}_{3} \mathrm{O}_{3} \mathrm{~N}_{2} \mathrm{Br}$.

La constitution de ce corps pouvait être interprétée de deux manières. Les produits intermédiaires, dans la réaction de Hofmann, ont une tendance très grande à la cyclisation ( $p$. ex. transformation de la phtal-diamide en benzoylène-urée) ${ }^{\mathbf{1}}$ ). Dans notre cas, on pouvait obtenir ainsi un dérivé de la p-diazine (II)

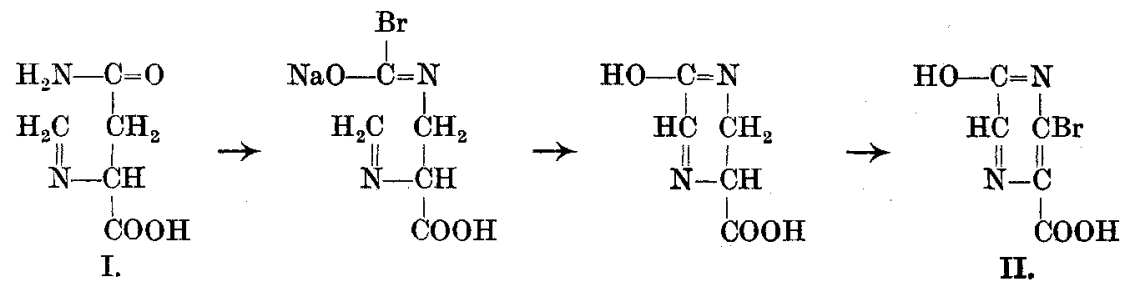

D'autre part, la cyclisation pouvait s'effectuer sans transposition intramoléculaire d'après le schéma suivant, qui conduirait à une m-diazine ou pyrimidine (III).

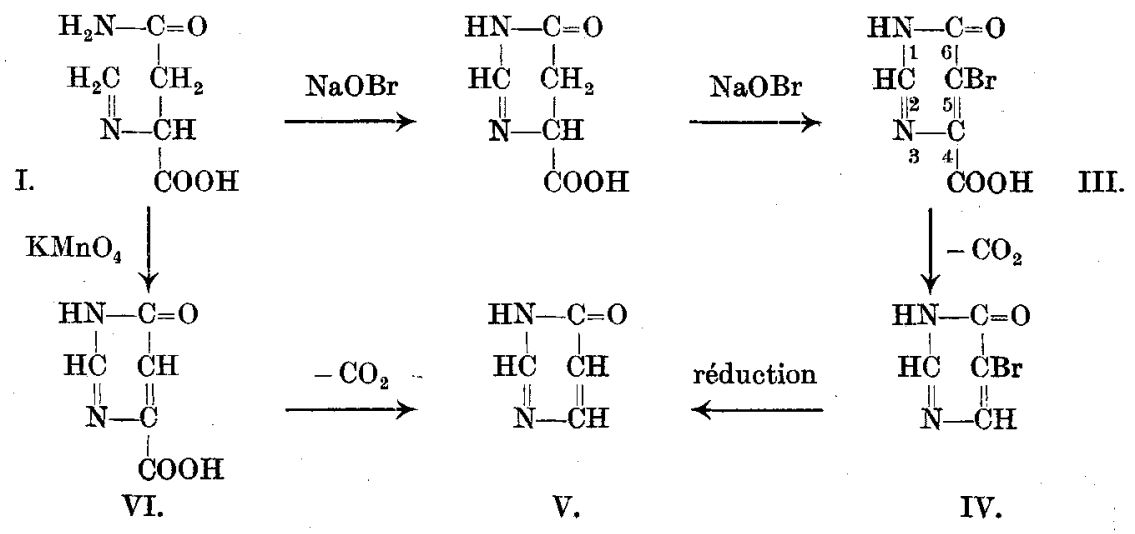

1) Hoogewerff, van Dorp, R. 15, 111 (1896). 
L'étude préliminaire de la réaction nous a montré qu'elle aboutit effectivement à l'acide 6-oxy-5-bromo-pyrimidine-4-carbonique (III). La présence du cycle pyrimidique dans ce corps est établie par les faits suivants. Porté à une température convenable, l'acide (III) se transforme en oxy-bromo-pyrimidine. (IV) par départ d'anhydride carbonique. Par réduction, cette base est transformée en un corps connu, la 6-oxy-pyrimidine (V). Nous parlerons plus loin de la position $\mathrm{du}$ brome.

L'hypobromite a donc agi à la fois comme oxydant (transformation de la méthylène-asparagine en dérivé dihydro-pyrimidique et pyrimidique) et comme halogénant. Nous n'avons toutefois pas pu isoler de produits intermédiaires; en présence d'une quantité insuffisante d'hypobromite on obtient le même acide, avec diminution proportionelle $d u$ rendement.

Cette même oxydation peut être réalisée avec le permanganate. On obtient dans ce cas, avec un rendement médiocre cependant, le corps non halogéné correspondant, l'acide 6-oxy-pyrimidine-4-carbonique (VI) transformé facilement par distillation en 6-oxy-pyrimidine (V).

Ces oxydations à l'hypobromite et au permanganate sont d'une application générale aux produits de condensation des aldéhydes avec le groupe amino de l'asparagine. Nous avons étudié les dérivés des aldéhydes formique, acétique et benzoïque. Le tableau suivant résume les réactions décrites dans cette communication.

Les acides bromés (1) obtenus par l'hypobromite se transforment vers $200^{\circ}$ en oxy-bromo-pyrimidines (2). Traitées par l'oxychlorure de phosphore, ces bases fournissent les chloro-bromo-pyrimidines (3). Par réduction à la poudre de zine, tous ces corps sont transformés en dérivés non halogénés correspondants $(4,5,6)$. Les oxy-acides (4) d'autre part sont obtenus directement par oxydation des aldéhydeasparagines au moyen du permanganate. Par départ d'anhydride carbonique ils se transforment en oxy-pyrimidines (5). Il est intéressant de constater que la substitution par le brome facilite le départ du groupe carboxyle.

Comme les produits de condensation avec les trois aldéhydes examinées fournissent des dérivés halogénés par l'action de l'hypobromite, le brome ne peut se trouver qu'en 1 ou en 5. L'hypobromite transforme les amides en dérivés bromés à l'azote, la position 1 pourrait par conséquent être prise en considération. La stabilité très grande des dérivés halogénés ne parle cependant pas en faveur de cette hypothèse: le sodium métallique n'attaque absolument pas l'oxy-bromo-pyrimidine 
en solution alcoolique bouillante. D'autre part, on peut bromer nos acides pyrimidine-carboniques par l'hypobromite: l'acide oxy-phénylcarbonique fournit avec ce réactif presque quantitativement un corps halogéné identique à celui obtenu par oxydation directe à l'hypobromite. Dans ce cas, il n'existe plus de groupe amino primaire particulièrement accessible à la substitution par l'halogène. On connaît du reste plusieurs cas de bromuration de pyrimidines en 5 par le brome en solution aqueuse neutre ${ }^{1}$ ) ou alcaline ${ }^{2}$ ).

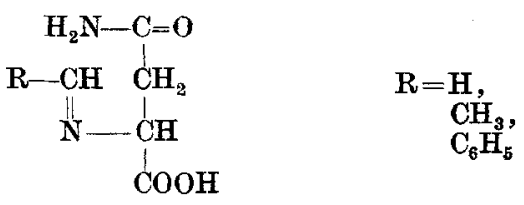

I.<smiles>[R]C1N=C(C(=O)O)C(Br)(Br)C(=O)N1</smiles>

2.<smiles>[R]C1=N[CH]C(Br)C1NC(=O)CBr</smiles><smiles>CC[Te]</smiles><smiles></smiles>

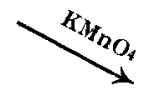<smiles>[R]c1nc(C(=O)O)cc(=O)[nH]c1=O</smiles>

4.

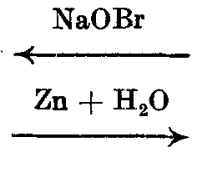<smiles>[Y]C(=O)[O-]</smiles>

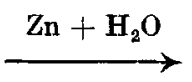<smiles>[R]C(=O)NC1[CH][CH]N=C1[R]</smiles>

5.<smiles>[R]C(CO)=NOC1COCCO1</smiles>

6.

La transformation de l'asparagine en dérivés pyrimidiques par oxydation en présence d'aldéhydes présente un intérêt particulier pour la biochimie végétale. Le rôle important des bases pyrimidiques et

1) R. Behrend, A. 229, 17 (1885): la 2,6-dioxy-4-méthyl-pyrimidine transformée en 2,6-dioxy-4-méthyl-5-bromo-pyrimidine.

2) O. Stark, A. 381, 152 (1911): la 2-oxy-4,6-diméthyl-pyrimidine trangformée en 2-oxy-4,6-diméthyl-5-bromo-pyrimidine. 
de leurs dérivés, les bases puriques, comme constituants des nucléoprotéides est connu. La synthèse de ces corps se fait probablement surtout dans les cellules végétales par un mécanisme dont on ignore encore tout. Dans le règne végétal, on trouve de l'asparagine en grandes quantités, surtout dans les jeunes pousses étiolées, c'est-à-dire dans des centres végétatifs placés dans des conditions d'assimilation entravée. C'est donc très probablement un produit intermédiaire du métabolisme des plantes. La réaction décrite établit un passage très simple de ce corps au cycle pyrimidique et pourra peut-être contribuer à en éclairer la genèse.

\section{OXYDATIONS A L'HYPOBROMITE.}

Nous avons effectué ces oxydations en additionnant à froid le produit de condensation de l'asparagine avec l'aldéhyde en solution alcaline, de 3 molécules d'hypobromite d'après l'équation:

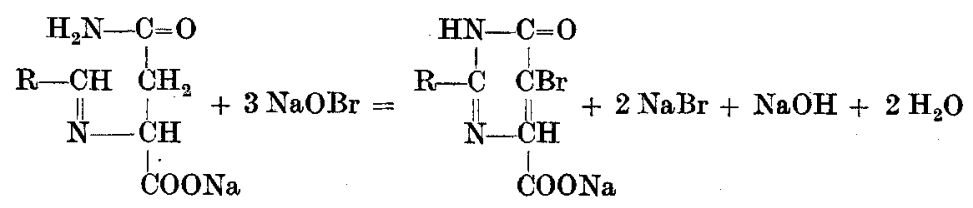

Les rendements ne sont bons que lorsque la solution d'hypobromite contient un grand excès d'alcali. Nous avons préparé notre oxydant en additionnant $125 \mathrm{~cm}^{3}$ de soude caustique $5-\mathrm{n}$. entre $-10^{0}$ et $-4^{0}$ de $30 \mathrm{gr}$. de brome en agitant bien. Le titrage par l'anhydride arsénieux montre que la formation d'hypobromite est quantitative. A la glacière, cette solution se conserve pendant quelques heures sans altération appréciable.

Acide 6-oxy-5-bromo-pyrimidine-4-carbonique.<smiles>O=C(O)c1ccccc1Br</smiles>

9 gr. de méthylène-asparagine préparée d'après $\left.S c h i f f^{1}\right)$ sont dissous à froid dans $50 \mathrm{~cm}^{3}$ d'eau et $12,50 \mathrm{~cm}^{3} \mathrm{NaOH} 5-\mathrm{n}$. A cette solution on ajoute goutte à goutte, en agitant et en refroidissant, une solution de 30 gr. de brome dans $125 \mathrm{~cm}^{3} \mathrm{NaOH} 5-n$. La réaction est

1) H. Schiff, A. 310, 30 (1899). 
accompagnée d'un dégagement perceptible de chaleur. Pendant cette opération qui dure environ 45 minutes, la température ne doit pas dépasser $-4^{0}$. Après repos d'une heure, on ajoute lentement la quantité d'acide chlorhydrique nécessaire pour neutraliser l'excès de soude caustique de l'hypobromite et pour mettre en liberté l'acide organique formé d'après l'équation donnée plus haut $\left(68,4 \mathrm{~cm}^{3} \mathrm{HCl} 5\right.$-n.). Il y a dégagement d'anhydride carbonique formé par suite de réactions secondaires, aveo production d'une mousse assez persistante. Vers la fin, l'acide organique commence à cristalliser. Après quelques heures, on filtre et lave à l'eau: rendement $9,5 \mathrm{gr}$. Les eaux-mères concentrées à 180 gr. (concentration à laquelle les sels inorganiques ne cristallisent pas encore) donnent encore 2,1 gr. de produit moins pur. Rendement total $11,6 \mathrm{gr}$. (78\%).

Par recristallisation dans de l'eau, on obtient de petites aiguilles incolores fondant à $206-207^{\circ}$ avec dégagement d'anhydride carbonique. L'acide est insoluble dans l'alcool, l'éther et le benzène, peu soluble dans l'eau froide, soluble dans 12 parties d'eau bouillante. La réaction de la solution aqueuse est acide au tournesol. Avec le nitrate d'argent, elle donne un précipité blane peu soluble dans l'eau chaude, soluble dans l'acide nitrique et l'ammoniaque d'où il est reprécipité par l'ébullition. L'acétate de cuivre précipite un sel neutre cristallisé, très peu soluble à froid.

Pour l'analyse, l'acide a été séché dans le vide sulfurique.

$0,1458 \mathrm{gr}$. de subst. ont donné 0,1343 gr. $\mathrm{CO}_{2}$ et 0,0317 gr. $\mathrm{H}_{2} \mathrm{O}$

0,1293 gr. de subst. ont donné $13,9 \mathrm{~cm}^{3} \mathrm{~N}_{2}\left(733 \mathrm{~mm}, 20^{\circ}, \mathrm{KOH} 1: 3\right)$

0,1826 gr. de subst. ont donné 0,0709 gr. $\mathrm{AgBr}$

0,3307 gr. de subst. à $110^{0}$ perdent 0,0253 gr. $\mathrm{H}_{2} \mathrm{O}$

$\mathrm{C}_{5} \mathrm{H}_{3} \mathrm{O}_{3} \mathrm{~N}_{2} \mathrm{Br}+\mathrm{H}_{2} \mathrm{O}$ Calculé C 25,33 H 2,12 N 11,82 Br 33,72 $\mathrm{H}_{2} \mathrm{O} \quad 7,60 \%$

Trouvé , 25,12, 2,42, $11,77,34,04,, 7,65 \%$

0,0926 gr. de subst. deshydratée à $110^{\circ}$ ont dégagé à $200^{\circ} 0,0191$ gr. $\mathrm{CO}_{2}$

$\mathrm{C}_{5} \mathrm{H}_{3} \mathrm{O}_{3} \mathrm{~N}_{2} \mathrm{Br}=\mathrm{C}_{4} \mathrm{H}_{3} \mathrm{ON}_{2} \mathrm{Br}+\mathrm{CO}_{2}$ Calculé $\mathrm{CO}_{2} 20,08 \%$

Trouvé , $20,60 \%$

Sel de cuivre: Les solutions chaudes de 0,25 gr. d'acide dans $5 \mathrm{~cm}^{3}$ d'eau et de $0,20 \mathrm{gr}$. d'acétate de cuirre dans $10 \mathrm{~cm}^{3}$ d'eau arec une goutte d'acide acétique glacial sont mélangées. La cristallisation du sel commence immédiatement. Il est filtré après refroidissement, lavé à l'eau et séché à $100^{\circ}$.

Cristaux microscopiques bleu clair, très peu solubles dans l'eau, perdant leur eau de cristallisation dans le vide $\grave{a} 100^{\circ}$ en se ternissant.

0,1656 gr. de subst. ont donné $0,0242 \mathrm{gr}$. $\mathrm{CuO}$

$\left(\mathrm{C}_{5} \mathrm{H}_{2} \mathrm{O}_{3} \mathrm{~N}_{2} \mathrm{Br}\right)_{2} \mathrm{Cu}+2 \mathrm{H}_{2} \mathrm{O}$ Calculé $\mathrm{Cu} 11,88 \%$

Trouvé , 11,70\% 
Ether éthylique: Obtenu en solution alcoolique par le gaz chlorhydrique. Le résidu huileux de la solution alcoolique neutralisée au carbonate de soude cristallise au contact de l'eau. Recristallisé dans l'eau.

Aiguilles incolores, fondant à $155^{\circ}-156^{\circ}$. Peu soluble dans l'eau. La solution aqueuse donne avec le nitrate d'argent un précipité soluble dans l'eau chaude, l'acide nitrique et l'ammoniaque.

0,1420 gr. de subst. ont donné 0,1767 gr. $\mathrm{CO}_{2}$ et $0,0409 \mathrm{gr}$. $\mathrm{H}_{2} \mathrm{O}$

$0,1245 \mathrm{gr}$. de subst. ont donné $12,7 \mathrm{~cm}^{3} \mathrm{~N}_{2}\left(730 \mathrm{~mm}, 19^{\circ}, \mathrm{KOH} 1: 3\right)$

$$
\begin{aligned}
& \mathrm{C}_{7} \mathrm{H}_{7} \mathrm{O}_{3} \mathrm{~N}_{2} \mathrm{Br} \text { Calculé C 34,01 } \mathrm{H} 2,85 \quad \mathrm{~N} 11,34 \% \\
& \text { Trouvé ,, 34,01 ,3,22 , 11,24\% }
\end{aligned}
$$

6-Oxy-5-bromo-pyrimidine.<smiles>O=C1N[CH+]C=NC1Br</smiles>

Le départ d'anhydride carbonique a lieu lorsqu'on porte l'acide à environ $200^{\circ}$. Dans le vide ou en présence d'un dissolvant, il a lieu sensiblement à la même température. Pour restreindre à un minimum la décomposition partielle que la base formée subit toujours, il convient d'opérer avec de petites quantités de substance pour pouvoir la porter rapidement à la température voulue, et d'éviter une surchauffe. La présence d'un corps indifférent, liquide à $200^{\circ}$, permet de réaliser ces conditions.

Un mélange de $1 \mathrm{gr}$. d'acide finement pulvérisé avec $1 \mathrm{gr}$. de naphtaline est plongé dans un bain porté à $220^{\circ}$. Le dégagement d'anhydride carbonique très rapide fait mousser le liquide. Dès que la réaction est terminée (disparition de la mousse), on verse le contenu brunâtre de l'éprouvette dans une capsule en porcelaine.

La masse pulvérisée est reprise avec $400 \mathrm{~cm}^{3}$ de benzène bouillant. La solution filtrée à chaud laisse déposer de petites paillettes blanches. Après 3 heures de repos, on filtre et lave au benzène. Les eaux-mères fortement concentrées laissent déposer un mélange de naphtaline et de base qu'on débarrasse de la naphtaline en le chauffant au bainmarie. Rendement 0,70 gr. (94\% de la théorie). Pour l'analyse, nous avons recristallisé le corps dans du xylène.

La base se présente sous forme de paillettes brillantes, du système orthorhombique, fondant à $197^{\circ}$. Flle est soluble, à l'ébullition, dans 250 parties de benzène, 120 parties de xylène, 10 parties d'alcool et 16 parties d'eau. 
Le chlorhydrate cristallise de la solution dans l'acide chlorhydrique concentré chaud en gros cristaux, fusibles à $206-207^{\circ}$, très solubles dans l'eau.

L'acide acétique glacial transforme la base en acétate assez soluble dans l'eau, fondant à $200-202^{\circ}$.

Le picrate fond à $150-151^{\circ}$.

La solution aqueuse de la base donne avec le nitrate d'argent un précipité microcristallin blanc, avec l'acétate de cuivre un précipité floconneux vert.

0,1289 gr. de subst. ont donné 0,1301 gr. $\mathrm{CO}_{2}$ et 0,0245 gr. $\mathrm{H}_{2} \mathrm{O}$

0,0712 gr. de subst. ont donné $10,65 \mathrm{~cm}^{3} \mathrm{~N}_{2}\left(724 \mathrm{~mm}, 19^{\circ}, \mathrm{KOH} 1: 3\right)$

0,1655 gr. de subst. ont donné $0,1802 \mathrm{gr}$. $\mathrm{AgBr}$

$\mathrm{C}_{4} \mathrm{H}_{3} \mathrm{ON}_{2} \mathrm{Br}$ Calculé C $27,45 \mathrm{H} \quad 1,72 \mathrm{~N} 16,02 \mathrm{Br} 45,67 \%$ Trouvé „, 27,50 „2,12,16,29, ,45,97\%

Picrate: Le mélange des solutions chaudes de $0,20 \mathrm{gr}$. de base dans $6 \mathrm{~cm}^{3}$ d'eau et de C 96 gr. d'acide picrique dans $15 \mathrm{~cm}^{3}$ d'eau donne par refroidissement 0,35 gr. de picrate. Recristallisé dans de l'alcool. Prismes microscopiques jaunes, fusibles à 150-1510.

0,1226 gr. de subst. ont donné $19,25 \mathrm{~cm}^{3} \mathrm{~N}_{2}\left(724 \mathrm{~mm}, 21^{0}\right.$, KOH $\left.1: 1\right)$

$\mathrm{C}_{20} \mathrm{H}_{6} \mathrm{O}_{3} \mathrm{~N}_{5} \mathrm{Br}$ Calculé $\mathrm{N} \quad 17,33 \%$

Trouvé , $17,35 \%$

6-Chloro-5-bromo-pyrimidine.

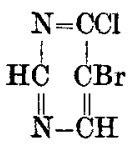

Dans un tube à. canon, on chauffe $5,5 \mathrm{gr}$. de base bromée avec $25 \mathrm{~cm}^{3}$ d'oxychlorure de phosphore 40 minutes à $140-145^{\circ}$. Le contenu liquide du tube est versé sur de la glace. La solution neutralisée au bicarbonate est épuisée à l'éther. L'extrait éthéré est distillé deux fois dans le vide. On obtient $3,10 \mathrm{gr}$. (51\% de la théorie) d'un liquide presqu'incolore passant sous $26 \mathrm{~mm}$ à $95,5^{0}$.

Liquide incolore, plus dense que l'eau, à odeur aromatique pénétrante, miscible aux dissolvants organiques, peu soluble dans l'eau. Picrate très peu soluble dans l'éther.

0,1107 gr. de subst. ont donné $0,0998 \mathrm{gr}$. $\mathrm{CO}_{2}$ et 0,0129 gr. $\mathrm{H}_{2} \mathrm{O}$

0,1217 gr. de subst. ont donné 0,1126 gr. $\mathrm{CO}_{2}$ et 0,0139 gr. $\mathrm{H}_{2} \mathrm{O}$

0,1503 gr. de subst. ont donné $19,8 \mathrm{~cm}^{3} \mathrm{~N}_{2}\left(733 \mathrm{~mm}, 24^{0}, \mathrm{KOH} 1: 1\right)$

0,1546 gr. de subst. ont donné $0,2645 \mathrm{gr}$. $\mathrm{AgCl}+\mathrm{AgBr}$

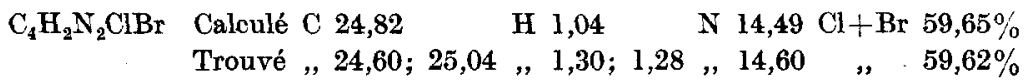


Ethylidène-asparagine.<smiles>CC(N=C([CH+]O)CC(C(N)=O)C(=O)O)C(N)=O</smiles>

On n'obtient ce corps avec un bon rendement que lorsqu'on opère la condensation en milieu légèrement alcalin, c'est-à-dire avec l'asparaginate de soude, en ayant soin d'évịter la présence d'alcali caustique libre.

10 gr. d'asparagine sont dissous dans $45 \mathrm{~cm}^{3}$ d'eau et $13,0 \mathrm{~cm}^{3}$ $\mathrm{NaOH} 5$-n. (théorie $13,30 \mathrm{~cm}^{3}$ ). Dans cette solution placée dans un mélange refrigérant, on introduit à $-8^{\circ}$, en agitant, $3 \mathrm{gr}$. d'aldéhyde acétique fraîchement distillée en un jet très mince. Il suffit de renfermer l'aldéhyde dans une ampoule étirée en un long tube capillaire dont on casse l'extrémité par une légère pression exercée contre l'agitateur. - La température monte un peu pendant cette opération.

Après repos d'une heure, on neutralise la soude caustique par $13,0 \mathrm{~cm}^{3} \mathrm{HCl} 5-\mathrm{n}$. Le liquide légèrement jaune laisse déposer peu à peu de petites aiguilles incolores, d'aspect nacré. Rendement 10,4 gr. (89\% de la théorie).

L'éthylidèné-asparagine ainsi obtenue cristallise avec une molécule d'eau et contient peut-être le groupement

$$
\begin{aligned}
-\mathrm{N}-\mathrm{C}- \\
\mathrm{H} \text { । }
\end{aligned}
$$

Elle est beaucoup moins stable que la méthylène-asparagine qui renferme vraiment le groupement méthylénique. Chauffée en tube ouvert, l'éthylidène-asparagine brunit et se décompose sans fondre comme l'asparagine. En tube fermé, elle fond à 230-2310. Par recristallisation dans l'eau chaude, déjà à $50^{\circ}$, on n'obtient que de l'asparagine. La solution dans l'eau froide (15 parties à $\left.20^{\circ}\right)$ acide au tournesol, sent l'aldéhyde acétique, preuve d'une dissociation partielle. Par concentration dans le vide, la solution aqueuse ne laisse cristalliser que difficilement un produit qui contient toujours de l'asparagine régénérée. L'éthylidène-asparagine est insoluble dans les dissolvants organiques. Les analyses sont faites avec le produit brut lavé à l'eau et séché sur du chlorure de calcium.

$0,2150 \mathrm{gr}$. de subst. ont donné $0,3179 \mathrm{gr}$. $\mathrm{CO}_{2}$ et $0,1311 \mathrm{gr} . \mathrm{H}_{2} \mathrm{O}$

0,1455 gr. de subst. ont donné $21,00 \mathrm{~cm}^{3} \mathrm{~N}_{2}\left(738 \mathrm{~mm}, 18^{\circ}, \mathrm{KOH} 1: 3\right)$

$$
\begin{aligned}
& \mathrm{C}_{6} \mathrm{H}_{10} \mathrm{O}_{3} \mathrm{~N}_{2}+\mathrm{H}_{2} \mathrm{O} \text { Calculé C 40,89 } \mathrm{H} \text { 6,87 N 15,91\% } \\
& \text { Trouvé , 40,33 ,6,82 , } 16,06 \%
\end{aligned}
$$


Acide 6-oxy-5-bromo-2-méthyl-pyrimidine-4-carbonique.<smiles>CCOC(=O)C1=NC(C)CC(=O)NC1=O</smiles>

Pour l'oxydation, il n'est pas nécessaire d'isoler l'éthylidèneasparagine.

La solution de $10 \mathrm{gr}$. d'asparagine condensés avec $3 \mathrm{gr}$. d'aldéhyde acétique est oxydée avec $30 \mathrm{gr}$. de brome en solution alcaline. Rendement en produit brut: $9,4 \mathrm{gr}$. (60\% de la théorie).

Par recristallisation dans l'eau, on obtient de petits cristaux incolores prismatiques fondant à $209-210^{\circ}$ avec dégagement de gaz. L'acide est insoluble dans les dissolvants organiques, soluble dans 15 parties d'eau bouillante. Ces propriétés le rapprochent absolument de l'acide oxy-bromo-carbonique.

1,3542 gr. de subst. séchée dans le vide sulfurique ont perdu à $120^{\circ} 0,0944 \mathrm{gr}$.

$$
\begin{aligned}
& \mathrm{C}_{6} \mathrm{H}_{5} \mathrm{O}_{3} \mathrm{~N}_{2} \mathrm{Br}+\mathrm{H}_{2} \mathrm{O} \text { Calculé } \mathrm{H}_{2} \mathrm{O} \quad 7,16 \% \\
& \text { Trouvé „ 6,96\% }
\end{aligned}
$$

$0,1417 \mathrm{gr}$. de subst. déshydratée ont donné $0,1614 \mathrm{gr}$. $\mathrm{CO}_{2}$ et $0,0280 \mathrm{gr}$. $\mathrm{H}_{2} \mathrm{O}$

0,1450 gr. de subst. ont donné $15,9 \mathrm{~cm}^{3} \mathrm{~N}_{2}\left(727 \mathrm{~mm}, 17^{\circ}, \mathrm{KOH} 1: 1\right)$

0,1519 gr. de subst. ont donné 0,1228 gr. $\mathrm{AgBr}$

$\mathrm{C}_{6} \mathrm{H}_{5} \mathrm{O}_{3} \mathrm{~N}_{2} \mathrm{Br}$ Calculé C 30,91 H·2,16 N 12,03 $\mathrm{Br} 34,30 \%$ Trouvé , 30,92 ,2,16 , 12,35 , $34,41 \%$

0,2899 gr. de subst. chauffés à $220^{\circ}$ ont donné 0,0550 gr. $\mathrm{CO}_{2}$

$$
\mathrm{C}_{6} \mathrm{H}_{5} \mathrm{O}_{3} \mathrm{~N}_{2} \mathrm{Br}=\mathrm{C}_{5} \mathrm{H}_{5} \mathrm{ON}_{2} \mathrm{Br}+\mathrm{CO}_{2} \text { Calculé } \mathrm{CO}_{2} \quad 18,88 \%
$$

Trouvé , 18,97\%

Sel de cuivre: Les solutions bouillantes mélangées de $0,30 \mathrm{gr}$. d'acide dans $5 \mathrm{~cm}^{3}$ d'eau et de $0,25 \mathrm{gr}$. d'acétate de cuivre dans $12 \mathrm{~cm}^{3}$ d'acide acétique très étendu laissent cristalliser de petits cristaux verts. - Lavé à l'eau, séché dans le vide sulfurique.

0,1124 gr. de subst. ont donné 0,0152 gr. CuO

$$
\begin{array}{r}
\left(\mathrm{C}_{6} \mathrm{H}_{4} \mathrm{O}_{3} \mathrm{~N}_{2} \mathrm{Br}\right)_{2} \mathrm{Cu}+2 \mathrm{H}_{2} \mathrm{O} \text { Calculé } \mathrm{Cu} 11,28 \% \\
\text { Trouvé , } 11,80 \%
\end{array}
$$

6-Oxy-5-bromo-2-méthyl-pyrimidine.<smiles>Cc1ncc(Br)c(=O)[nH]1</smiles>

Préparée par décomposition de l'acide oxy-bromo-méthyl-pyrimidine-carbonique comme l'oxy-bromo-pyrimidine, la nouvelle base se 
précente sous forme de prismes longs, brillants, fondant à $231-232^{\circ}$, solubles à l'ébullition dans 125 parties de benzène, 80 parties de xylène, 70 parties d'éther, 8,5 parties d'alcool et 16 parties d'eau.

Chlorhydrate très soluble dans l'eau, fondant à $229^{\circ}-230^{\circ}$ avec décomposition.

Acétate assez soluble dans l'eau fondant à $234^{\circ}$.

Picrate fondant à $163^{\circ}-164^{\circ}$.

Sel d'argent blanc, assez soluble dans l'eau chaude, sel de cuivre (par l'acétate de cuivre) vert floconneux.

$0,1827 \mathrm{gr}$. de subst. ont donné $0,2115 \mathrm{gr}$. $\mathrm{CO}_{2}$ et $0,0409 \mathrm{gr}$. $\mathrm{H}_{2} \mathrm{O}$

0,1046 gr. de subst. ont donné $13,85 \mathrm{~cm}^{3} \mathrm{~N}_{2}\left(725 \mathrm{~mm}, 22^{\circ}, \mathrm{KOH} 1: 1\right)$

0,1219 gr. de subst. ont donné 0,1213 gr. AgBr

$$
\begin{aligned}
& \mathrm{C}_{5} \mathrm{H}_{5} \mathrm{ON}_{2} \mathrm{Br} \text {. Calculé C 31,76 H 2,17 N } 14,82 \text { Br 42,29\% } \\
& \text { Trouvé , 31,58 , 2,51 , 14,16 , 42,34\% }
\end{aligned}
$$

Picrate: par addition de la solution chaude de $0,20 \mathrm{gr}$. de base dans $5 \mathrm{~cm}^{3}$ d'eau à 0,25 gr. d'acide picrique dans $15 \mathrm{~cm}^{3} \mathrm{~d}^{\prime}$ eau et concentration au bain-marie.

Recristallisé dans de l'alcool, aiguilles jaunes fondant à $163^{\circ}-164^{0}$.

0,1265 gr. de subst. ont donné $19,2 \mathrm{~cm}^{3} \mathrm{~N}_{2}\left(730 \mathrm{~mm}, 2^{\circ}, \mathrm{KOH} 1: 1\right)$

$$
\begin{array}{r}
\mathrm{C}_{11} \mathrm{H}_{8} \mathrm{~N}_{5} \mathrm{O}_{3} \mathrm{Br} \quad \begin{array}{c}
\text { Calculé N } 16,76 \% \\
\text { Trouvé , } 16,80 \%
\end{array}
\end{array}
$$

\section{6-Chloro-5-bromo-2-méthyl-pyrimidine.}<smiles>Cc1nnc(Cl)c(Br)n1</smiles>

1,5 gr. d'oxy-bromo-méthyl-pyrimidine traités par $7 \mathrm{~cm}^{3}$ d'oxychlorure de phosphore comme pour la préparation de la chloro-bromopyrimidine donnent $0,80 \mathrm{gr}$. (48\% de la théorie) de chloro-bromométhyl-pyrimidine.

Liquide incolore, plus dense que l'eau, à odeur aromatique pénétrante distillant à $107,5^{\circ}$ sous $27 \mathrm{~mm}$. Miscible aux dissolvants organiques, très peu soluble dans l'eau. Picrate très peu soluble dans l'éther.

$0,1278 \mathrm{gr}$. de subst. ont donné $0,1368 \mathrm{gr}$. $\mathrm{CO}_{2}$ et $0,0255 \mathrm{gr} . \mathrm{H}_{2} \mathrm{O}$

$0,1580 \mathrm{gr}$. de subst. ont donné $19,3 \mathrm{~cm}^{3} \mathrm{~N}_{2}\left(730 \mathrm{~mm}, 22^{\circ}, \mathrm{KOH} 1: 3\right)$

0,2055 gr. de subst. ont donné $0,3263 \mathrm{gr}$. $\mathrm{AgCl}+\mathrm{AgBr}$

$$
\begin{aligned}
& \mathrm{C}_{5} \mathrm{H}_{4} \mathrm{~N}_{2} \mathrm{ClBr} \text { Calculé C 28,94 } \mathrm{H} \text { 1,94 N 13,50 } \mathrm{Cl}+\mathrm{Br} 55,62 \% \\
& \text { Trouvé ,, 29,18 , 2,23 , 13,24 , 55,34\% }
\end{aligned}
$$


Acide 6-oxy-5-bromo-2-phényl-pyrimidine-4-carbonique.

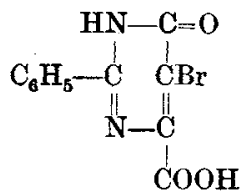

Agitée avec une solution d'asparaginate de soude, l'aldéhyde benzoïque s'y dissout à peu près en quantité équimoléculaire. Le produit de condensation est encore moins stable que l'éthylidène-asparagine. La solution acidulée par l'acide carbonique se trouble immédiatement par régénération de l'aldéhyde. Par concentration dans le vide ou par précipitation avec de l'alcool, on n'obtient que des sirops cristallisant très difficilement. Comme on obtient les produits d'oxydation de la benzylidène-asparagine en opérant avec sa solution alcaline, nous avons renoncé à l'isoler.

10 gr. d'asparagine dans $50 \mathrm{~cm}^{3}$ d'eau et $13,0 \mathrm{~cm}^{3}$ de soude caustique 5-n. sont seconés avec $7,3 \mathrm{gr}$. d'aldéhyde benzoïque (théorie 7,1 gr.) pendant 4 heures. L'aldéhyde a presque complètement disparu. Le liquide clair obtenu par filtration sur un filtre mouillé est oxydé par $30 \mathrm{gr}$. de brome en solution alcaline.

Le produit brut est souillé d'un peu d'acide benzoïque qu'on élimine par une chauffe à l'étuve à $110^{\circ}$. Le résidu, légèrement coloré et cristallisant mal, a été purifié par précipitation lente de sa solution alcaline filtrée. Rendement en produit pur 6,9 gr. (environ $38 \%$ de la théorie).

Petites aiguilles blanches fondant à $250-252^{\circ}$ sans dégagement de gaz, très peu solubles dans l'eau bouillante $(1 / 900)$, l'alcool bouillant $(1 / 250)$, insolubles dans le benzène, le xylène, l'éther.

La fusion sans dégagement de gaz par une élévation lente de la température s'explique par le départ d'anhydride carbonique avant la fusion. Ce point de fusion de $252^{\circ}$ est par conséquent celui de l'oxybromo-phényl-pyrimidine résultant de la décomposition de l'acide. Plongé dans un bain à $260^{\circ}$, l'acide fond avec dégagement de gaz. Le départ d'anhydride carbonique se fait déjà à partir de $130^{\circ}$.

0,0860 gr. de subst. ont donné 0,1442 gr. $\mathrm{CO}_{2}$ et 0,0186 gr. $\mathrm{H}_{2} \mathrm{O}$

0,1099 gr. de subst. ont donné $9,8 \mathrm{~cm}^{3} \mathrm{~N}_{2}\left(732 \mathrm{~mm}, 18^{\circ}, \mathrm{KOH} 1: 3\right)$

$$
\begin{aligned}
& \mathrm{C}_{11} \mathrm{H}_{7} \mathrm{O}_{3} \mathrm{~N}_{2} \mathrm{Br} \text { Calculé C 44,76 H 2,39 N 9,50\% } \\
& \text { Trouvé „, 45,01 , } 2,41 \quad, 9,86 \%
\end{aligned}
$$


6-Oxy-5-bromo-2-phényl-pyrimidine.<smiles>CCc1ncc(Br)c(=O)[nH]1</smiles>

En chauffant l'acide à l'étuve à $130-140^{\circ}$ à poids constant (env. 30 heures) on obtient la base avec un rendement presque quantitatif. Recristallisé pour l'analyse dans du benzène.

Longues aiguilles prismatiques, d'aspect soyeux, fondant à $\mathbf{2 5 2}^{\mathbf{0}}$, solubles à l'ébullition dans 110 parties d'alcool, 80 parties de benzène, 60 parties de xylène, très peu solubles dans l'eau froide $(1 / 12000)$.

0,1155 gr. de subst. ont donné 0,2024 gr. $\mathrm{CO}_{2}$ et 0,0301 gr. $\mathrm{H}_{2} \mathrm{O}$

0,1206 gr. de subst. ont donné $11,85 \mathrm{~cm}^{3} \mathrm{~N}_{2}\left(730 \mathrm{~mm}, 19^{\circ}, \mathrm{KOH} 1: 1\right)$

0,1286 gr. de subst. ont donné $0,0958 \mathrm{gr}$. $\mathrm{AgBr}$

$$
\begin{aligned}
& \mathrm{C}_{10} \mathrm{H}_{7} \mathrm{ON}_{2} \mathrm{Br} \text { Calculé } \mathrm{C} 47,82 \quad \mathrm{H} \quad 2,81 \text { N } 11,16 \quad \mathrm{Br} 31,84 \% \\
& \text { Trouvé , 47,80 ,2,91 ,11,07 ,31,70\% }
\end{aligned}
$$

6-Chloro-5-bromo-2-phényl-pyrimidine.

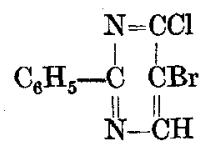

4 gr. d'oxy-bromo-phényl-pyrimidine fournissent avec $16 \mathrm{~cm}^{3}$ d'oxychlorure de phosphore 2,6 gr. (43\% de la théorie) d'un produit passant à la seconde distillation à $193,5^{\circ}$ sous $24 \mathrm{~mm}$ et cristallisant immédiatement.

Longues aiguilles prismatiques à reflets nacrés, incolores, fondant à $130-131^{\circ}$, solubles dans les dissolvants organiques, presque insolubles dans l'eau.

0,1497 gr. de subst. ont donné $13,85 \mathrm{~cm}^{3} \mathrm{~N}_{2}\left(732 \mathrm{~mm}, 22^{\circ}, \mathrm{KOH} \mathrm{l}: 1\right)$

0,1323 gr. de subst. ont donné $0,1620 \mathrm{gr}$. $\mathrm{AgCl}+\mathrm{AgBr}$

$$
\begin{aligned}
& \mathrm{C}_{10} \mathrm{H}_{6} \mathrm{~N}_{2} \mathrm{ClBr} \text { Calculé } \mathrm{N} 10,39 \mathrm{Cl}+\mathrm{Br} 42,82 \% \\
& \text { Trouvé , } 10,32 \quad, \quad 42,70 \%
\end{aligned}
$$

2-Phényl-pyrimidine.

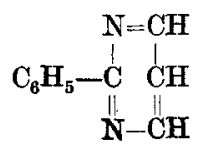

$6 \mathrm{gr}$. de chloro-bromo-phényl-pyrimidine suspendus dans $600 \mathrm{~cm}^{3}$ d'eau sont chauffés à reflux avec $60 \mathrm{gr}$. de poudre de zinc débarrassée 
d'oxyde par lavage à l'acide chlorhydrique dilué. Après 4 heures d'ébullition on distille la phényl-pyrimidine à la vapeur d'eau. La base cristallise en grande partie dans le réfrigérant.

Reprise à l'éther et distillée dans le vide, elle passe à $157,5^{\circ}$ sous $25 \mathrm{~mm}$ en se solidifiant rapidement. Rendement $1,6 \mathrm{gr}$. (46\% de la théorie).

Pour l'analyse, nous avons recristallisé ce produit légèrement violacé du point de fusion $126-127^{\circ}$ dans 18 parties d'éther de pétrole bouillant. Paillettes blanches, très légères, fondant à $128^{\circ}$, solubles à chaud dans l'éther de pétrole, l'éther, le benzène d'où elles cristallisent avec du benzène, solubles à froid dans l'éther acétique. Les solutions se colorent facilement en violet par oxydation. Soluble à froid dans l'acide chlorhydrique. L'acide chlorhydrique concentré chaud résinifie la base.

0,1292 gr. de subst. ont donné 0,3630 gr. $\mathrm{CO}_{2}$ et $0,0572 \mathrm{gr}$. $\mathrm{H}_{2} \mathrm{O}$

$$
\begin{array}{rrr}
\mathrm{C}_{10} \mathrm{H}_{8} \mathrm{~N}_{2} & \begin{array}{c}
\text { Calculé C } 76,89 \\
\text { Tronvé ,, 76,63 }
\end{array} \\
& \text {, 4,16\% } \\
& 4,96 \%
\end{array}
$$

Picrate: $0,10 \mathrm{gr}$. de phényl-pyrimidine dans $4 \mathrm{~cm}^{3}$ d'éther de pétrole chaud sont additionnés de $0,16 \mathrm{gr}$. d'acide picrique dans de l'éther. La solution se colore en rouge foncé. Après évaporation de l'éther au bain-marie, la solution laisse déposer des cristaux brun foncé peu solubles dans l'éther de pétrole bouillant, très solubles dans l'éther. Recristallisé dans un mélange d'éther et d'éther de pétrole $(1+5)$.

Cristaux microscopiques bruns, fondant à $108^{\circ}$.

0,1544 gr. de subst. ont donné $25,1 \mathrm{~cm}^{3} \mathrm{~N}_{2}\left(730 \mathrm{~mm}, 24^{\circ}, \mathrm{KOH} \mathrm{l:1}\right)$

$$
\begin{aligned}
& \mathrm{C}_{16} \mathrm{H}_{11} \mathrm{O}_{7} \mathrm{~N}_{5} \begin{array}{l}
\text { Calculé } \mathrm{N} 18,18 \% \\
\text { Trouvé ;, } 17,94 \%
\end{array}
\end{aligned}
$$

\section{OXYDATIONS AU PERMANGANATE.}

Nous oxydons l'aldéhyde-asparagine dissoute dans un équivalent d'alcali par addition fractionnée de la quantité théorique de permanganate de potassium $(4 \mathrm{H})$ en solution à $5 \%$ en maintenant la température à environ $15^{\circ}$. La décoloration est assez rapide. Après repos d'une nuit, le bioxyde de manganèse formé d'abord à l'état colloïdal s'est déposé. Pour l'agglomérer encore davantage, on chauffe au bainmarie, on filtre et reprend le précipité plusieurs fois par l'eau bouillante pour en retirer la substance organique adsorbée.

Les filtrats additionnés de la quantité d'acide chlorhydrique nécessaire pour transformer tout l'alcali en chlorure laissent déposer après concentration les acides oxy-pyrimidine-carboniques souillés toujours de sels inorganiques. 
Les rendements sont en thèse générale assez médiocres, surtout pour les premiers termes de la série où les cristallisations nécessaires pour obtenir des produits sans cendres entraînent des pertes considérables. Par l'augmentation de la quantité de permanganate, le rendement n'est pas amélioré.

Acide 6-oxy-pyrimidine-4-carbonique.

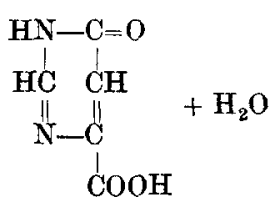

a) Par oxydation au permanganate.

10 gr. de méthylène-asparagine dans $69,5 \mathrm{~cm}^{3}$ de soude caustique normale sont oxydés par 14,75 gr. de permanganate de potasse.

Les filtrats réunis acidulés par $32,4 \mathrm{~cm}^{3} \mathrm{HCl}$ 5-n. abandonnent après concentration $1,1 \mathrm{gr}$. de produit du point de fusion $268-270^{\circ}$ de l'acide pur, mais contenant des sels inorganiques. Par cristallisations répétées dans de l'eau on obtient finalement $0,30 \mathrm{gr}$. de produit exempt de cendres mais dont l'analyse, après dessiccation dans le vide sulfurique, n'a pas donné de très bons résultats.

Prismes microscopiques, fondant à $268-270^{\circ}$ avec dégagement de gaz, solubles dans 15 parties d'eau bouillante d'où ils cristallisent très lentement, insolubles dans l'alcool, l'éther et le benzène.

La solution aqueuse donne avec l'acétate de cuivre un précipité bleu verdâtre, avec le nitrate d'argent un précipité microcristallin blanc, très peu soluble dans l'eau à l'ébullition.

0,0935 gr. de subst. ont donné 0,1333 gr. $\mathrm{CO}_{2}$ et $0,0272 \mathrm{gr} . \mathrm{H}_{2} \mathrm{O}$

0,1111 gr. de subst. ont donné $17,85 \mathrm{~cm}^{3} \mathrm{~N}_{2}\left(731 \mathrm{~mm}, 23^{\circ}\right.$, NOH $\left.1: 1\right)$

$$
\begin{array}{rrrrr}
\mathrm{C}_{5} \mathrm{H}_{4} \mathrm{O}_{3} \mathrm{~N}_{2}+\mathrm{H}_{2} \mathrm{O} & \text { Calculé } \mathrm{C} 38,02 & \mathrm{H} & 3,79 & \mathrm{~N} 17,72 \% \\
& \text { Trouvé , } 38,80 & , 3,25 & , 17,80 \%
\end{array}
$$

b) Par réduction de l'acide oxy-bromo-pyrimidine-carbonique.

1 gr. d'acide bromé dans $120 \mathrm{~cm}^{3}$ d'eau est chauffé à reflux pendant 3 heures avec $10 \mathrm{gr}$. de poudre de zinc.

Le liquide filtré est débarrassé du zine par l'hydrogène sulfuré. Après filtration et addition d'acide chlorhydrique, on évapore à sec. Le résidu dissous dans $2 \mathrm{~cm}^{3}$ d'eau est additionné de $7 \mathrm{~cm}^{3}$ d'alcool absolu. Un précipité floconneux se dépose qui se transforme après 36 
heures de repos en prismes microscopiques. La solution de ce produit dans peu d'eau laisse déposer lentement des prismes microscopiques, fondant à $267^{\circ}$ avec dégagement de gaz, ne contenant plus d'halogène.

\section{6-Oxy-pyrimidine.}<smiles>O=C1CCCN1</smiles>

a) Par décomposition de l'acide oxy-pyrimidine-carbonique.

0,8 gr. d'acide sont distillés dans le vide. On obtient un produit jaunâtre qui passe à $210^{\circ}$ sous $12 \mathrm{~mm}$ et qui se solidifie immédiatement au contact des parties froides du ballon. On dissout le distillat dans $400 \mathrm{~cm}^{3}$ d'éther bouillant. Par concentration jusqu'au commencement de la cristallisation on obtient 0,20 gr. de longues aiguilles incolores, fondant à $160-161^{\circ}$. Redissous dans $300 \mathrm{~cm}^{3}$ d'éther et concentré à $20 \mathrm{~cm}^{3}$ : cristallisation brusque de $0,15 \mathrm{gr}$. de base correspondant aux indications bibliographiques ${ }^{1}$ ) à l'exception du point de fusion qui est de $161^{\circ}$ au lieu de $164-165^{\circ}$.

$$
\begin{aligned}
& 0,1115 \text { gr. de subst. ont donné } 0,2038 \text { gr. } \mathrm{CO}_{2} \text { et } 0,0488 \text { gr. } \mathrm{H}_{2} \mathrm{O} \\
& \mathrm{C}_{4} \mathrm{H}_{4} \mathrm{ON}_{2} \text { Calculé } \mathrm{C} 49,98 \text { H } 4,19 \\
& \text { Trouvé ,, } 49,85 \quad, 4,76
\end{aligned}
$$

b) Par réduction de l'oxy-bromo-pyrimidine.

3,5 gr. de base bromée dans $350 \mathrm{~cm}^{3}$ d'eau sont réduits par 3 heures d'ébullition avec 35 gr. de poudre de zinc. Le liquide débarrassé du zinc par l'hydrogène sulfuré est additionné d'acide chlorhydrique et concentré au bain-marie à $45 \mathrm{~cm}^{3}$.

Après neutralisation par le carbonate de potasse on reprend à l'éther qui laisse un petit résidu incolore, cristallisé, fondant à $163^{\circ}$ à $164^{\circ}$, ne contenant pas d'halogène.

Le picrate recristallisé dans l'éther fond à $189^{\circ}$ au lieu de $190^{\circ}$ ).

1) H. L. Wheeler, C. 1907 II. 1529.

2) loc. cit. 
Acide 6-oxy-2-méthyl-pyrimidine-4-carbonique.<smiles>Cc1nc(C(=O)O)cc(=O)[nH]c1=O</smiles>

10 gr. d'asparagine condensés avec $3 \mathrm{gr}$. d'aldéhyde acétique et oxydés avec 14'gr. de permanganate de potasse donnent 3,4 gr. de produit brut (fondant à $243^{\circ}$ ). Après 6 cristallisations dans l'eau on obtient 1,1 gr. de produit pur, fondant à $261^{01}$ ) avec dégagement de gaz, retenant dans le vide sulfurique deux molécules d'eau. L'acide est soluble dans 14 parties d'eau bouillante, insoluble dans les dissolvants organiques.

Le sel de cuivre précipité par l'acétate de cuivre est de composition variable. Chauffé avec de l'oxyde de cuivre, l'acide donne un sel basique cristallisé bleu.

0,1362 gr. de subst. ont donné 0,1880 gr. $\mathrm{CO}_{2}$ et 0,0699 gr. $\mathrm{H}_{2} \mathrm{O}$

$$
\begin{array}{lll}
\mathrm{C}_{6} \mathrm{H}_{6} \mathrm{O}_{3} \mathrm{~N}_{2}+2 \mathrm{H}_{2} \mathrm{O} & \begin{array}{l}
\text { Calculé C 37,80 } \\
\text { Trouvé , } 5,68 \%
\end{array} \\
& \text { Th,65 } & 5,74 \%
\end{array}
$$

Sel de cuivre basique, séché dans le vide sulfurique

$$
\begin{array}{cc}
0,0651 \text { gr. ont donné } 0,0227 \text { gr. CuO } \\
\mathrm{C}_{6} \mathrm{H}_{5} \mathrm{O}_{3} \mathrm{~N}_{2} \cdot \mathrm{CuOH} \quad \begin{array}{l}
\text { Calculé } \mathrm{Cu} 27,23 \% \\
\text { Trouvé , } 27,86 \%
\end{array}
\end{array}
$$

$$
\begin{aligned}
& \text { 6-Oxy-2-méthyl-pyrimidine. } \\
& \begin{array}{r}
\mathrm{HN}-\mathrm{C}=\mathrm{O} \\
\mathrm{J} \\
\mathrm{CH}_{3}-\mathrm{C} \quad \mathrm{CH} \\
\mathrm{N}-\mathrm{CH}
\end{array}
\end{aligned}
$$

0,5 gr. d'acide oxy-méthyl-pyrimidine-carbonique sont distillés dans le vide. Sous $22 \mathrm{~mm}$ il passe entre $200^{\circ}$ et $215^{\circ} 0,25 \mathrm{gr}$. d'un produit jaunâtre qui se solidifie immédiatement. Recristallisé deux fois dans $500 \mathrm{~cm}^{3}$ d'éther par concentration à $30 \mathrm{~cm}^{3}$, on obtient des aiguilles incolores $d u$ point de fusion constant de $208^{\circ}$ avec suintement, inférieur au point de fusion $212^{\circ}$ de la bibliographie ${ }^{2}$ ). Chauffé dans

1) A. Pinner, B. 25, 1423 (1892), a préparé ce corps. II indique que l'acide deshydraté est stable à $300^{\circ}$, ce qui est en contradiction avec nos observations et avec le fait que tous les acides pyrimidine-carboniques à groupe carboxyle voisin d'un azote se décomposent avec départ d'anhydride carbonique au-dessous de $300^{\circ}$.

2) S. Gabriel, B. 37, 3640 (1904). 
le vide à $70^{\circ}$, le corps est débarrassé d'une impureté qui donne une sublimation cristalline, et fond alors à $212^{\circ}$.

0,0792 gr. de subst. ont donné 0,1588 gr. $\mathrm{CO}_{2}$ et $0,0412 \mathrm{gr}$. $\mathrm{H}_{2} \mathrm{O}$

$$
\begin{array}{rrr}
\mathrm{C}_{5} \mathrm{H}_{6} \mathrm{ON}_{2} & \text { Calculé C 54,52 } & \mathrm{H} 5,49 \% \\
& \text { Trouvé „, 54,66 } & \mathbf{5 , 8 3} \%
\end{array}
$$

Acide 6-oxy-2-phén yl-pyrimidine-4-carbonique.<smiles></smiles>

9,35 gr. d'asparagine condensés avec 6,8 gr. d'aldéhyde benzoïque sont oxydés par 13,2 gr. de permanganate de potasse. Le produit brut est séché à $120^{\circ}$ pour éliminer un peu d'acide benzoïque. Par reprécipitation de sa solution alcaline on obtient $3,45 \mathrm{gr}$. de produit incolore, ne contenant pas de cendres ( $26 \%$ de la théorie).

Cristaux microscopiques fondant à $247^{\circ}$ avec dégagement de gaz ${ }^{1}$ ), solubles à chaud dans 750 parties d'eau, 43 parties d'acide acétique glacial, 44 parties de benzoate d'éthyle, insolubles dans l'éther, le benzène, le xylène, l'éther acétique.

0,0764 gr. dé subst. ont donné $9,2 \mathrm{~cm}^{3} \mathrm{~N}_{2}\left(729 \mathrm{~mm} ., 18^{\circ}, \mathrm{KOH} 1: 1\right)$

$$
\begin{array}{r}
\mathrm{C}_{11} \mathrm{H}_{8} \mathrm{O}_{3} \mathrm{~N}_{2} \text { Calculé } \mathrm{N} 12,96 \% \\
\text { Trouvé ", } 13,26 \%
\end{array}
$$

Sel de cuivre: 0,15 gr. d'acide dans $5 \mathrm{~cm}^{3} \mathrm{NaOH}$ 0,2-n. sont additionnés de $0,38 \mathrm{gr}$. de sulfate de cuivre oristallisé dans $4 \mathrm{~cm}^{3}$ d'eau. Après concentration et refroidissement on recueille de petits cristaux verts. Séché dans le vide sulfurique.

0,0877 gr. de subst. ont donné $0,0144 \mathrm{gr}$. $\mathrm{CuO}$

$\left(\mathrm{C}_{11} \mathrm{H}_{7} \mathrm{O}_{3} \mathrm{~N}_{2}\right)_{2} \mathrm{Cu}+2 \mathrm{H}_{2} \mathrm{O}$ Calculé Cu $12,88 \%$

Trouvé ,, $13,11 \%$

L'acide cristallisant très mal, nous l'avons transformé pour le dosage du carbone et de l'hydrogène en son éther éthylique obtenu par traitement de l'acide suspendu dans de l'alcool absolu contenant du gaz chlorhydrique. Par concentration de la solution neutralisée, on obtient des aiguilles incolores du point de fusion $84-85^{\circ}$ qui ne change pas par recristallisation dans de l'alcool.

0,1053 gr. de subst. ont donné 0,2481 gr. et 0,0492 gr. $\mathrm{H}_{2} \mathrm{O}$

$$
\begin{aligned}
& \mathrm{C}_{13} \mathrm{H}_{12} \mathrm{O}_{3} \mathrm{~N}_{2} \text { Calculé C 63,91 H 4,95\% } \\
& \text { Trouvé „ 64,27 , 5,23\% }
\end{aligned}
$$

2) A. Pinner, B. 22, 1629 (1889). 
Bromuration de l'acide oxy-phényl-pyrimidine-4-carbonique: acide 6-oxy2-phényl-5-bromo-4-carbonique.

1 gr. d'acide non bromé dans $23 \mathrm{~cm}^{3}$ de soude caustique $0,2-\mathrm{n}$. est additionné à $-10^{\circ}$ de $0,74 \mathrm{gr}$. de brome dans $18,6 \mathrm{~cm}^{3}$ de soude caustique normale.

On acidule avec $18,6 \mathrm{~cm}^{3}$ d'acide normal. Le produit qui se dépose est purifié par précipitation de sa solution alcaline: póint de fusion $252^{\circ}$, rendement $1,25 \mathrm{gr}$. (92\% de la théorie).

0,1329 gr. de subst. ont donné $0,0822 \mathrm{gr}$. $\mathrm{AgBr}$

$\mathrm{C}_{11} \mathrm{H}_{7} \mathrm{O}_{3} \mathrm{~N}_{2} \mathrm{Br}$ Calculé $\mathrm{Br} 27,09 \%$

Trouvé , 27,12\%

0,30 gr. d'acide décomposés par la chaleur donnent un produit qui cristallisé dans du benzène fond à $252^{\circ}$ de même que son mélange avec la 6-oxy-2-phényl-5-bromo-pyrimidine.

$$
\begin{array}{r}
\text { 6-Oxy-2-phényl-pyrimidine. } \\
\mathrm{HN}-\mathrm{C}=\mathrm{O} \\
\stackrel{\mathrm{C}}{\mathrm{C}} \\
\mathrm{C}_{8} \mathrm{H}_{5}-\mathrm{CH} \\
\mathbb{N}-\mathrm{CH}
\end{array}
$$

Par distillation de $1 \mathrm{gr}$. d'acide oxy-phényl-pyrimidine-carbonique dans le vide, à $12 \mathrm{~mm}$, on obtient $0,60 \mathrm{gr}$. de base passant vers $245^{\circ}$. Le produit jaune qui n'est pas décoloré par le noir animal, est recristallisé dans du benzène en présence d'oxyde de cuivre en poudre.

On obtient ainsi de fines aiguilles incolores, point de fusion $206^{\circ}$ à $207^{\circ}$ (au lieu de $207-208^{\circ}$ ), conformes à la bibliographie ${ }^{1}$ ). Séché dans le vide sur de la paraffine.

0,0953 gr. de subst. ont donné 0,2442 gr. $\mathrm{CO}_{2}$ et $0,0415 \mathrm{gr}$. $\mathrm{H}_{2} \mathrm{O}$

$\mathrm{C}_{10} \mathrm{H}_{8} \mathrm{ON}_{2}$ Calculé $\mathrm{C} 69,75 \quad \mathrm{H} 4,68 \%$

Trouvé „, 69,88 • "4,81\%

Picrate: 0,10 gr. de base dans $6 \mathrm{~cm}^{3}$ d'alcool à chaud sont additionnés de $0,18 \mathrm{gr}$. d'acide picrique dans $7 \mathrm{~cm}^{3}$ d'alcool chaud. Par refroidissement, il cristallise $0,15 \mathrm{gr}$. de picrate qui sont recristallisés dans de l'alcool.

Aiguilles jaunes fondant à $151^{\circ}$.

0,0936 gr. de subst. ont donné $14,40 \mathrm{~cm}^{3} \mathrm{~N}_{2}\left(733 \mathrm{~mm}, 20^{\circ}, \mathrm{KOH} \mathrm{1:1)}\right.$

$$
\begin{aligned}
& \mathrm{C}_{16} \mathrm{H}_{11} \mathrm{O}_{8} \mathrm{~N}_{5} \text { Calculé } \mathrm{N} \quad 17,46 \% \\
& \text { Trouvé , 17,17\% }
\end{aligned}
$$

Genève, Laboratoire de Chimie organique de l'Université.

1) A. Pinner, B. 22, 2617 (1889). - S. Ruhemann, A. S. Hemmy, B. 30, 1491 (1897). 